\title{
Gravity Wave Momentum Flux in Directional Shear Flows over Three- dimensional Mountains: Linear and Nonlinear Numerical Solutions as Compared to Linear Analytical Solutions
}

\author{
Xin $\mathrm{Xu}^{1,2}$, Ming Xue ${ }^{2,3,1}$, and Yuan Wang ${ }^{1}$ \\ ${ }^{1}$ Key Laboratory of Mesoscale Severe Weather/Ministry of Education and School of \\ Atmospheric Sciences, Nanjing University, Nanjing, 210093, China \\ ${ }^{2}$ Center for Analysis and Prediction of Storms and ${ }^{3}$ School of Meteorology, \\ University of Oklahoma, Norman Oklahoma 73072
}

Submitted to J. of Geophysical Research

October, 2012

Final version April 30, 2013

Corresponding author address

Ming Xue

Center for Analysis and Prediction of Storms and School of Meteorology, University of Oklahoma, Norman Oklahoma 73072

Email: $\underline{\text { mxue@ou.edu }}$ 


\begin{abstract}
Gravity waves forced by circular bell-shaped mountains are simulated using the Advanced Regional Prediction System (ARPS) model for four directionally sheared wind profiles W1, W2, W2a and W2b. W1 is eastward at the surface while the latter three are southeastward. With the vertical shear directed northward, W1 shows an increasing speed with height, whereas W2, W2a and W2b are first decelerated in the bottom layer (beneath different heights). Gravity wave momentum flux (WMF) and its vertical divergence at four different Froude numbers $(\mathrm{Fr})$ are investigated. At a large Froude number $\mathrm{Fr}=80$ where the waves are essentially linear, the simulated WMF and WMF divergence agree well with their linear analytic counterparts, thus providing a numerical validation for the analytical solutions of $\mathrm{Xu}$ et al. For moderately nonlinear waves at $\mathrm{Fr}=1.6$, the simulated WMFs are qualitatively similar to the analytical values but are amplified due to nonlinear effect. For more nonlinear waves at lower Froude numbers, an enhanced drag is found at the lower $F r=0.4$ in W1, W2a and W2b, and a low drag is found at higher Fr = 0.8 in W2, in contrast to the corresponding constant-flow solutions. Analyses on the mountain flow structure show that the height variation of the ambient wind speed has an important influence on the airflow behavior (e.g., flow over versus flow around a mountain), which in turn affects the drag. Thus, unlike the constant-flow case, the wavedrag state in turning flows cannot be determined from the surface-based Froude number alone and new wave drag parameterization schemes would be needed properly account for directional shear effects. Additionally, directional wind shear can also introduce asymmetric development in the wake flow to trigger lee vortex shedding, which is observed in W2 at $\mathrm{Fr}=0.4$.
\end{abstract}




\section{Introduction}

Mountain gravity waves can exert considerable forcing on the overlying atmospheric flow when significant vertical divergences exist with the horizontal wave momentum that they carry; such flux divergence can occur when wave breaking occurs or when directional turning exists with the mean flow over orographic forcing. Recently, Xu et al. [2012, XWX12 hereafter] studied the solutions of the vertical flux of horizontal momentum, or wave moment flux (WMF) for short, associated with gravity waves that develop over a three-dimensional (3D) obstacle in stably stratified flows turning directionally with height. Using linear, hydrostatic wave theory, general expressions were derived for the WMF and its vertical divergence, and special cases with idealized wind profiles over circular bell-shaped mountains were examined.

Nevertheless, linear theory only applies when Froude number $\mathrm{Fr}>>1$ where $\mathrm{Fr}=$ $|\mathbf{V}| /\left(N h_{0}\right)$ with $|\mathbf{V}|$ being the upstream wind speed, $N$ the Brunt-Väisälä frequency and $h_{0}$ the mountain height. At low Froude number of order unity, the airstream undergoes a transition from "flow-over" to "flow-around" regime, as a consequence of wave-breaking induced bifurcation on the windward slope [Smith and Grønås, 1993]. Dramatic deviations from linear solutions have been found in the flow response at low Froude numbers, including the development of lee side vortices [Hunt and Snyder, 1980; Smolarkiewicz and Rotunno, 1989], lee vortex streets [Etling, 1989; Sun and Chern, 1994; Schär and Durran, 1997; Vosper, 2000], and potential vorticity (PV) banners [Aebischer and Schär, 1998; Jiang et al., 2003; Schär et al., 2003; Grubišić, 2004; Wang and Tan, 2009].

Miranda and James [1992, hereafter MJ92] studied the dependency of WMF on Fr, suggesting a "high-drag" state (compared to the wave drag obtained by linear wave theory) approximately in the range $0.5<\mathrm{Fr}<1$ but a "low-drag" state when $0.2<\mathrm{Fr}<$ 0.5. Eckermann et al. [2010] obtained qualitatively similar results by investigating the WMF in flows of variable Froude number. The "high-drag" is attributed either to wave reflection from a critical level aloft [Clark and Peltier, 1984; Miranda and Valente, 1997], or to "hydraulic jump" of airflow beneath a well-mixed turbulent layer [Smith, 1985]. Both mechanisms highlight the important role of wave breaking above the mountain [Durran and Klemp, 1987]. The "low-drag" at small Froude number is fundamentally due to lateral flow splitting, which largely reduces the wave amplitudes aloft [Smith, 1989].

Earlier studies focused almost exclusively on orographic waves generated in unidirectional flows. However, realistic winds often change their speed and direction with height. It has been demonstrated recently that the turning of the wind with height has a pronounced influence on the WMF [XWX12 and references therein]. Teixeira and Miranda [2009] addressed the WMF numerically in vertically turning flows but their study was limited to weakly nonlinear waves only, with a lower $\mathrm{Fr}$ limit of 2. More nonlinear waves within directionally turn flows and their relationship to corresponding linear solutions have not been investigated in the literature so far. Dörnbrack et al. [2005] simulated a directional shear flow above a bell-shaped mountain but focusing mainly on the reliability of numerical model solutions.

In this study, we examine numerically the WMF in directional shear flows for a wider range of Froude numbers, using the Advanced Regional Prediction System [ARPS, Xue et al., 2000] model and the solutions are compared to linear analytical solutions 
obtained in $X W X 12$. The study also serves to validate the model numerical solutions for flow regimes that they should agree with linear solutions. The remainder of this paper is organized as follows. The design of numerical experiments is described in section 2 . Section 3 validates the model solutions by comparing the simulated WMF at large Froude number to that obtained from linear wave theory. The WMF and the flow structure at low Froude numbers are presented and analyzed in section 4, with conclusions given in section 5 .

\section{Setup of numerical experiments}

The ARPS is a 3D, fully compressible, non-hydrostatic atmospheric model developed at the Center for Analysis and Prediction of Storms (CAPS), the University of Oklahoma. Governing equations are formulated in generalized terrain-following coordinates and solved using finite differences on Arakawa C-grid. Mode-splitting time integration technique is employed to improve the model efficiency. Readers are referred to Xue et al. [2000] for a complete description of the dynamical and numerical framework of ARPS, and for verifications of two-dimensional mountain wave solutions. The ARPS had also been applied to large amplitude mountain wave problems, including related model inter-comparisons [Doyle et al., 2000; 2011]. In these model intercomparison studies, the ARPS tended to produce solutions close to the mean of the model ensemble, suggesting the trustworthiness of its solutions for orographic flows.

For all numerical experiments, the horizontal grid interval is $\Delta x=\Delta y=3 \mathrm{~km}$ but with variable domain length $L$ (see Table 1). The model domain depth $\left(H_{0}\right)$ is $16 \mathrm{~km}$ for most experiments, but is reduced to 6 or $8 \mathrm{~km}$ for stronger shear profiles (W2a and W2b, see Table 2) since the wind speed can become excessively large at the upper levels in such cases. A Rayleigh damping layer is placed above $H_{0} / 2$, with the damping coefficient carefully tuned to minimize wave reflection from the model top. Below the damping layer, the model has a quasi-uniform (quasi because of the non-flat terrain) resolution of $\Delta z=50 \mathrm{~m}$ which is stretched vertically in the damping layer. Wave radiation boundary conditions are employed at the top and side boundaries. The 1.5-order turbulent kinematic energy (TKE) closure scheme is used for subgrid-scale turbulence mixing, accompanied with weak 4th-order horizontal computational mixing to damp small-scale numerical noise. The Earth's rotation, surface fluxes and radiation physics are neglected. Moisture related model options are also switched off as we are dealing with purely dry processes. Free slip boundary conditions are applied at the lower boundary.

The model is initialized by an ambient flow impinging upon a circular bell-shaped mountain given by

$$
h(x, y)=h_{0}\left\{1+\left[\left(x-x_{c}\right) / a\right]^{2}+\left[\left(y-y_{c}\right) / a\right]^{2}\right\}^{-3 / 2},
$$

where $a=20 \mathrm{~km}$ is the mountain half width, $\left(x_{c}, y_{c}\right)=(360,720) \mathrm{km}$ is the location of mountain summit and $h_{0}$ the maximum mountain height. Given the mountain width, the 3 $\mathrm{km}$ grid spacing used is found to be sufficient to accurately resolve the wave structures and WMF; improvement is within a few percent with further increase in resolution (not shown). For the ambient flow, two directional shear winds examined in XWX12 are adopted (W1 and W2, see Table 2) which vary linearly with height,

$$
\mathbf{V}(z)=\mathbf{V}_{0}+\mathbf{V}_{z}=\left(U_{0}+U_{z} z, V_{0}+V_{z} z\right) \text {. }
$$


They have the same vertical shear of $\mathbf{V}_{z}=\left(0,5 \times 10^{-3}\right) \mathrm{s}^{-1}$ and surface speed $\left|\mathbf{V}_{0}\right|=8 \mathrm{~m} \mathrm{~s}^{-1}$ but have different surface wind directions. W1 is eastward at the surface with $\mathbf{V}_{0}=(8,0)$ $\mathrm{m} \mathrm{s}^{-1}$ whereas W2 is directed to southeast such that $\mathbf{V}_{0}=(4 \sqrt{2},-4 \sqrt{2}) \mathrm{m} \mathrm{s}^{-1}$. Two additional directional shear winds, W2a and W2b, are also considered which are the same as W2 but have much stronger vertical shears of $\mathbf{V}_{z}=\left(0,10^{-2}\right) \mathrm{s}^{-1}$ and $\mathbf{V}_{z}=\left(0, \sqrt{2} \times 10^{-2}\right)$ $\mathrm{s}^{-1}$, respectively. Among these wind profiles, the speed of $\mathrm{W} 1$ keeps increasing with height, but W2, W2a and W2b are firstly decelerated until $1.13 \mathrm{~km}, 0.57 \mathrm{~km}$ and $0.4 \mathrm{~km}$ height, respectively. Moreover, a constant wind, W0 with $\mathbf{V}_{0}=(8,0) \mathrm{m} \mathrm{s}^{-1}$, is considered as a reference. Assuming a constant Brunt-Väisälä frequency of $N=0.01 \mathrm{~s}^{-1}$, the dimensionless mountain width is $N a /\left|\mathbf{V}_{0}\right|=25$, indicating that the mean flow should be more or less in hydrostatic balance. The model is run until the wave fields reach a steady state, if it exists.

Before investigating the directionally sheared wind case, the case of constant wind W0 is examined. Four experiments (ExpCST1 to ExpCST4 in Table 1) are performed with mountain heights ranging from $h_{0}=0.01 \mathrm{~km}$ to $2 \mathrm{~km}$, corresponding to surface-based Froude numbers of $F r=80,1.6,0.8$, and 0.4 . Figure 1 shows the constantflow WMFs scaled by their analytic counterparts given by

$$
\left|\mathbf{F}_{0}\right|=0.25 \pi \bar{\rho} N a h_{0}^{2}\left|\mathbf{V}_{0}\right| \text {, }
$$

at non-dimensional time $T=\left|\mathbf{V}_{0}\right| t / a=72$. Gravity waves forced by a low mountain with $h_{0}=10 \mathrm{~m}(\mathrm{Fr}=80)$ are essentially linear. The simulated WMF accounts for about $98 \%$ of the linear theory prediction at the surface. It decreases slightly with height by $\sim 5 \%$ at $z$ $=8 \mathrm{~km}$, immediately below the Rayleigh damping layer. Such small discrepancies $(<5 \%)$ between the model and linear theory solutions are typical of numerical solutions of mountain waves over low mountains; Xue et al. [2000] showed about 3\% error for WMFs of a constant flow over a $1 \mathrm{~m}$ mountain using the ARPS.

For a moderate mountain with $h_{0}=500 \mathrm{~m}(F r=1.6)$, the model also maintains the WMF with height very well, but the WMF is about $10 \%$ greater than its linear counterpart, due to nonlinear effect [MJ92]. The simulated WMF for a 1-km-high mountain $(F r=0.8)$ has the largest amplification among the three constant-flow cases, yielding a WMF that is $31 \%$ higher than the corresponding linear theory prediction at the mountain top. However, it decreases a little more notably with height, showing a reduction of about $9 \%$ at $z=8 \mathrm{~km}$. For an even higher mountain with $h_{0}=2 \mathrm{~km}(\mathrm{Fr}=$ 0.4 ), the model generates a much smaller (normalized) WMF that accounts for only about $29 \%$ of the corresponding linear solution at the mountain top; this is a result of significant lateral flow splitting (less flow is forced over the mountain top) at such a low Froude number [MJ92].

In general, the constant-flow WMFs obtained here show a similar dependency on the surface-based Froude number as in MJ92 and Eckermann et al. [2010], in spite of the differences in the numerical models used (e.g. ARPS versus WRF) and in the experiment setup (e.g., domain size, model resolution, airflow speed, and mountain width). The agreement with previous studies gives us a basis to investigate the more complex, nonconstant-flow, cases. 


\section{Linear wave experiments}

In order to validate the ability of the above model configuration in accurately simulating 3D mountain waves in directional shear flows, some linear wave experiments are conducted with the maximum mountain height $h_{0}$ set to $0.01 \mathrm{~km}$, corresponding to $\mathrm{Fr}$ $=80$. The linear experiments include Exp1, Exp2, Exp2a, Exp2b, and two variations of Exp1 (Exp1a and Exp1b) where the domain width is varied to test sensitivity to model size.

For linear, hydrostatic waves in flows of the type given by Eq. (2) over a circular bell-shaped orography, the WMF, F, and its vertical divergence, $\mathbf{L}$, are given by Eqs. (25) and (26) in $X W X 12$ and reproduced here as

$$
\begin{aligned}
& \mathbf{F}(z)=0.5 \bar{\rho} N a h_{0}^{2}\left|\mathbf{V}_{0}\right| \int_{\varphi_{L}}^{\varphi_{U}}(\cos \varphi, \sin \varphi) \cos \left(\psi_{0}-\varphi\right) \sqrt{1-\cos ^{2}\left(\chi_{0}-\varphi\right) /(4 \mathrm{Ri})} d \varphi, \\
& \mathbf{L}(z)= \pm\left[\mathbf{k} \times \frac{\mathbf{V}(z)}{|\mathbf{V}(z)|}\right] 0.5 \bar{\rho} N^{2} a h_{0}^{2}\left|\mathbf{V}_{0}\right| \frac{\sin \left|\psi(z)-\psi_{0}\right|}{|\mathbf{V}(z)|} \sqrt{1-\left\{1-\frac{\sin ^{2}\left[\chi_{0}-\psi(z)\right]}{2 \mathrm{Ri}}\right\}^{2},}
\end{aligned}
$$

where $\bar{\rho}$ is the constant reference density, $\chi_{0}$ the azimuth of the vertical shear, $\psi_{0}$ and $\psi(z)$ the wind azimuths at the surface and height $z$, and $\mathrm{Ri}=N^{2} /\left|\mathbf{V}_{z}\right|^{2}$ is the ambient flow Richardson number (Ri). For backing winds, the lower and upper limits of the integral in Eq. (4) are $\varphi_{L}=\psi(z)-\pi / 2$ and $\varphi_{U}=\psi_{0}+\pi / 2$, whilst $\varphi_{L}=\psi_{0}-\pi / 2$ and $\varphi_{U}=\psi(z)+\pi / 2$ for veering winds. The plus and minus signs in Eq. (5) are applied for backing and veering winds, respectively. WMF and its vertical divergence in directionally sheared winds were also derived by Teixeira and Miranda [2009] by using the WKB method. The WKB method is strictly only valid for slow-varying winds at high Ri. However, Teixeira and Miranda [2009] found that the WKB solution can be valid for a Ri as low as order unity.

Figure 2 shows the vertical profiles of the magnitude of simulated WMFs in the linear wave experiments at non-dimensional time $T=43.2$, at which the wave fields have reached steady state. Also shown are the linear wave theory results (solid lines) according to Eq. (4). All WMFs are scaled by the momentum flux of linear hydrostatic waves of corresponding constant flow with the same surface wind speed given by Eq. (3). As seen from Fig. 2, the WMFs decrease with height, which is owing to selective critical level absorption [Shutts, 1995; XWX12], i.e., for different wave components, the height of the critical level is different. The simulated WMFs are in general close to their analytic counterparts, even at a low Richardson number of $\mathrm{Ri}=0.5$ (Fig. 2d). Note that the WMF is over predicted by about $3.8 \%$ at this low $\mathrm{Ri}$, whereas the model tends to underestimate the WMF at relatively higher Ri of order unity (see Figs. 2a, 2b and 2c). The difference between the numerical and analytic values is about $2-5 \%$ at the surface, increasing to $\sim 14 \%, 9 \%$ and $10 \%$ at the level right below the damping layer in Exp1, Exp2 and Exp2a, respectively. By contrast, the model WMF in Exp2b agrees better with its analytic counterpart, especially between $z=0.4 \mathrm{~km}$ and $z=1 \mathrm{~km}$ (Fig. 2d).

Similar discrepancy had also been noted in previous studies [e.g., Teixeira and Miranda, 2009] and the discrepancy is primarily attributed to lateral spreading of 3D gravity waves as well as numerical errors. As studied by Smith [1980] and Shutts [1998], the wave pattern widens with height such that more wave activities will propagate out of the computational domain at higher altitudes. To examine the impact of the lateral boundary of a limited model domain, we ran experiments Exp1a and Exp1b (Table 1) whose domain width are 1.25 and 1.5 times that of Exp1 in the $y$ direction, respectively. 
As can be seen in Fig. 2a, the simulated WMFs agree with the linear theory prediction somewhat better as the domain size increases; the error at $8 \mathrm{~km}$ height is reduced from about $14 \%$ to about $9 \%$ in Exp1b as the domain is 50\% larger; still the solution with the default width is not too far off, especially at the lower levels. Given the high computational cost of running the model with grid sizes on the order of $500 \times 500 \times 200$ points for extended lengths, the default domain width of $L_{0}=1440 \mathrm{~km}$ is deemed acceptable and used in all other experiments.

Figure 3 displays the vertical profiles of the WMF vertical divergence for these linear wave experiments. The numerical solutions also show rather good agreement with the linear wave theory. The altitudes where the WMF divergence peaks are well simulated although the amplitudes of the simulated WMF divergence are underestimated by up to $23 \%$, larger than the WMF error itself. The large WMF divergence error has to do with the fact the divergence is a derivate of the WMF itself, which can be more sensitive to the vertical resolution of the model, among other factors.

The numerical solutions for low mountains (high Froude number) from the above experiments agree at least qualitatively with the analytical linear wave solutions; indicating that model with the given configurations can serve as an effective tool for studying more nonlinear gravity waves forced by 3D mountains in directional shear flows; here the use of a sufficiently large model domain is key as our earlier simulations with much smaller domains produced far inferior results.

\section{Nonlinear wave experiments}

\subsection{Wave momentum flux}

A number of nonlinear wave experiments are conducted with the mountain height $h_{0}=0.5 \mathrm{~km}, 1 \mathrm{~km}$ and $2 \mathrm{~km}$, respectively. Their Froude numbers are thus $\mathrm{Fr}=1.6,0.8$ and 0.4. Figure 4 presents the vertical profiles of the normalized WMFs at $T=100.8$. Note that, unlike other experiments, the wave fields in Exp8 do not attain a steady state by $T=100.8$, the reason of which will be discussed later.

In the case of $\mathrm{Fr}=1.6$ (open circles in Fig. 4), the simulated WMFs are greater than those predicted by linear wave theory due to nonlinear effect. At $z=0.5 \mathrm{~km}$ (i.e., at the mountain peak), the WMF shows an increase of about 9\% in Exp3 (Fig. 4a with W1 wind profile), similar to the amplification of the constant-flow WMF at the same surfacebased Froude number (Fig. 1), while it is enhanced much more notably by about 33\% in Exp4 (Fig. 4b with W2 wind profile), 23\% in Exp4a (Fig. 4c with W2a wind profile), and $22 \%$ in Exp4b (Fig. 4d with W2b wind profile). At Fr $=0.8$ (solid circles), the WMFs in Exp5 (Fig. 4a), Exp6a (Fig. 4c) and Exp6b (Fig. 4d) exhibit a drag enhancement as in the corresponding constant flow case (Fig. 1), being about 25\%, 7\% and 19\% larger than the corresponding analytical values at $z=1 \mathrm{~km}$. On the contrary, a "low-drag” WMF is found in Exp6 using the W2 wind profile (Fig. 4b), accounting for only about $86 \%$ of the corresponding linear value. At even lower Froude number of $\mathrm{Fr}=0.4$ (triangles), the numerical model also produces WMFs of opposite (high versus low) drag states for these turning wind profiles. At $z=2 \mathrm{~km}$ (again the level of mountain peak), the simulated WMF accounts for only about 27\% of the linear theory prediction in Exp8 for wind profile W2 (Fig. 4b). In contrast, WMFs at the same height are increased by $\sim 30 \%, 19 \%$, and as high as 110\% in Exp7 (Fig. 4a), Exp8a (Fig. 4c), and Exp8b (Fig. 4d), respectively. 
The WMFs also change with height differently at low Froude numbers. Figure 5 shows the vertical divergence profiles of the WMFs. At $F r=1.6$ (open circles) the simulated WMFs have similar height variations as their analytic counterparts. While the amplitudes of the WMF divergences in Exp3 (Fig. 5a), Exp4a (Fig. 5c), and Exp4b (Fig. 5d) are underestimated, it is slightly overestimated in Exp4 (Fig. 5b). When Fr $=0.8$ (solid circles), Exp5 (Fig. 5a), Exp6a (Fig. 5c) and Exp6b (Fig. 5d) show nearly the same WMF divergence as in Exp3, Exp4a and Exp4b, respectively, yet the flux divergence has much smaller values than the linear theory values between $1 \mathrm{~km}$ and $4 \mathrm{~km}$ in Exp6 (Fig. 5b). At $\mathrm{Fr}=0.4$ (triangles), the WMF divergence is much smaller than the linear theory prediction in Exp8 (Fig. 5b) while the over-predictions by the linear theory in Exp7 (Fig. 5a), Exp8a (Fig. 5c) and Exp8b (Fig. 5d) are much less. There are also some irregularities in the height variations with the numerical solution.

Because of the distinct behaviors of the WMFs and their vertical divergences at the low Froude numbers, the linear theory predictions appear to be unreliable.

\subsection{Flow structures}

The WMF is closely linked to the flow response to the orographic forcing. While the WMFs for these flow profiles (W1, W2, W2a and W2b) are both enhanced at Fr = 1.6, they are in opposite drag states at $F r=0.8$ and 0.4 , however. Structures of the mountain flows at the latter two Froude numbers are examined next. Figure 6 shows the surface wind vectors and Fig. 7 shows the isentropes in the vertical cross-sections in the direction of surface winds through the mountain peak for these nonlinear wave experiments.

At $\mathrm{Fr}=0.8$, the incident flows for these wind profiles can readily go over the mountain (Figs. 7a, 7b, 7c and 7d), with weak horizontal deflection (Figs. 6a, 6b, 6c and $6 d)$. No stagnation point forms on the windward slope of the mountain. Over the downwind slope, the airflow is accelerated in Exp5, Exp6a and Exp6b (Figs. 6a, 6c, and $6 \mathrm{~d}$ ) and the isentropes are gently displaced in the vertical without overturning (Figs. 7a, 7c, and 7d), similar to the case of large Froude numbers [cf. Fig. 3a of Smolarkiewicz and Rotunno, 1989]. On the contrary, significantly decelerated flows and weak recirculations are found in the mountain wake in Exp6 (Fig. 6b), in association with steep isentropes on the downwind slope (Fig. 7b).

Nevertheless, the isentropes in all cases are weakly disturbed at the upper levels, namely, the waves are of small amplitudes aloft (not shown). This can also be inferred from Fig. 4 that the WMF decays with height. In addition to the small wave amplitudes, the directional flow has high speeds at the high altitudes. As a consequence, the formation of flow stagnation and hence breakdown of gravity waves aloft [Smith, 1989], which is critical to the "high-drag" state at low Froude number in constant flows [Durran and Klemp, 1987; MJ92; Eckermann et al., 2010], tend to be inhibited in the turning flow environment.

At $\mathrm{Fr}=0.4$, the low-level flow does not have enough kinetic energy to overcome the potential energy barrier of the mountain. There is therefore prominent lateral flowsplitting along with a stagnation point on the windward slope of the mountain (Figs. 6e and 6f) or on its right flank (right when facing downstream) (Figs. 6g and 6h). Taking Exp7 as an example, the upstream flow begins to split at $x \approx-2 a$, accompanied with flow reversal from $x \approx-0.7 a$ (the origin is at the mountain peak). However, flow splitting is more notable in Exp8 than in Exp7, Exp8a and Exp8b at the same Froude number. 
As shown in Fig. 7e, overturning flows are mainly confined below $\sim 0.6 \mathrm{~km}$ in Exp7. The incident flow originating above $\sim 1.3 \mathrm{~km}$ height at the upstream is capable of going over the $2 \mathrm{~km}$ mountain. Similar behaviors are found for the airflows in Exp8a and Exp8b (Figs. 7g and 7h). Behind the mountain peak, the flow descends considerably over the lee slope and thus generates strong downslope winds at the expense of its potential energy (Figs. 6e, 6g, 6h). These strong downslope winds prevent the low-level deflected fluids from flowing into the wake. In consequence, there is no flow recirculation downwind of the mountain. The lee vortices that would have occurred in a constant flow of the same surface-based Froude number [Hunt and Snyder, 1980] do not form in our case of directionally turning winds.

In Exp8, only upstream fluids above $1.7 \mathrm{~km}$ can flow over the mountain top (Fig. $7 \mathrm{f}$ ). Upwind flow reversal takes place at as high as $\sim 1.2 \mathrm{~km}$. Meanwhile, a wake region is well developed behind the mountain, characterized by relatively quiescent fluids and salient flow recirculation (Fig. 6f). Furthermore, the wake flow pattern is asymmetric with respect to the incident flow at the surface, contrasting to that in constant flows. According to Schär and Smith [1993b] and Schär and Durran [1997], lee vortex shedding will be triggered in a low-Froude number flow through the development of asymmetric perturbations which can destroy the wake stability. This issue will be addressed in next section.

\subsection{Lee vortex shedding}

The wave fields in Exp8 are not steady at $T=100.8$, as mentioned in Section 4.1. The unsteady feature is evident from the temporal evolution of the surface pressure drag. According to Fig. 8, the pressure drag is not stabilized even the model is run up to $T=$ 172.8. Instead, it evolves to an oscillating state after a rapid initial decrease in the early time. Such drag oscillations occurring at low Froude number were attributed to the transient lee vortex shedding by Schär and Durran [1997] and Vosper [2000].

Figure 9 depicts the time evolution of vertical vorticity at the surface in Exp8. At an earlier time of $T=7.2$ (Fig. 9a), the leeside vortices at the inner edge of the split flow branches remain attached to the mountain. Later at $T=14.4$ (Fig. 9b), they have been shed from the mountain and are drifting downstream. Meanwhile, new vortices are created on the flanks of the mountain. Later at $T=43.2$ (Fig. 9f), the vortices formed earlier have exited the plotting domain. As the time proceeds, lee vortices are continuously produced, distorted and shed off, forming a vortex street which is akin to those observed behind large mountains and islands in the atmosphere [Etling, 1989, and references therein]. A comparison experiment Exp8U similar to Exp8 is performed (see Table 1), which uses wind profile W2U; W2U has the same wind speed profile as the W2 used in Exp8 but does not change direction with height. In this unidirectional wind situation, the wake flow reaches a steady state rather than transiting into a state with vortex shedding (not shown).

Sun and Chern [1994] have suggested several effects of asymmetry responsible for vortex shedding, such as rotation, asymmetric mountain shape, inclination of the wind to the mountain, and asymmetric perturbations superposed upon the mean flow. Here it is demonstrated that the directional wind shear can also promote lee vortex shedding, a factor that had not been considered before. Detailed formation mechanisms of lee vortex shedding in the directional shear wind circumstance can be investigated in future studies. 


\subsection{Discussions}

From the previous subsections, the mountain flow structures at low Froude numbers are affected by the directional wind shear significantly, which in turn alters the WMF. Such behaviors appear to be closely linked to the height variation of the ambient wind speed up to the mountain top level. For the W1 profile having an increasing speed with height, it is easier for the flow to climb over the mountain. In contrast, the flow with the W2 profile is prone to go around the mountain as the flow is actually decelerated with height (the surface negative flow in the $y$ direction decreases to zero then reverses direction with height) in the bottom layer below about $1.1 \mathrm{~km}$. For strongly sheared wind profiles W2a and W2b, although their speeds are also decreased with height first, the deceleration is confined below $0.57 \mathrm{~km}$ and $0.4 \mathrm{~km}$. Above this shallow layer, their speeds start to increase rapidly with height. Therefore W2a and W2b can also readily go over the high mountain.

The difference between the WMFs tends to increase as the Froude number lowers (as the mountain height increases or as the flow speed decreases). For example, at $\mathrm{Fr}=$ 0.4 the model generates a WMF in W1 (Exp7) that is almost 4 times greater than that in W2 (Exp8). At Fr = 1.6, the discrepancy between the two WMFs is no more than $30 \%$. This is apparently because when the flow speed is marginal for it to climb over the mountain, the details in its vertical variations below the mountain peak can dramatically change the flow behavior, including flow over versus flow around the mountain, hence significantly affecting the resulting WMF.

In view of the dramatically different flow structure and WMF even at the same surface-based Froude number, it is no longer appropriate to determine the drag state in turning flows merely based upon the Froude number at the surface. This should have important implications on the parameterization of low-level mountain wave drag in numerical models [Kim et al., 2003]. Current low-level wave drag parameterization schemes [Kim and Arakawa, 1995; Lott and Miller, 1997; Gregory et al., 1998; Scinocca and McFarlane, 2000; Kim and Doyle, 2005], where the drag state is typically controlled by the surface-based Froude number, are thus not suitable for the directionally sheared flow case.

Moreover, the presence of the directional wind shear means the pre-existence of horizontal vorticity in the ambient flow. This base state horizontal vorticity can contribute to the formation of vertical vorticity through vertical tilting, in addition to the baroclinically generated horizontal vorticity [Smolarkiewicz and Rotunno, 1989; Rotunno et al., 1999]. Their relative contributions will require detailed diagnostics to quantify.

Another issue of great interest is the creation of PV in the mountain wake. While the vertical vorticity can be generated inviscidly and adiabatically, the generation of PV in an initially PV-free flow depends on dissipative processes that lead to Bernoulli function deficit in the wake [Schär, 1993]. Surface friction and internal turbulence caused by wave-breaking or flow-splitting were found to contribute towards PV creation [Schär and Smith, 1993a; Thorpe et al., 1993; Grubišić et al., 1995; Schär and Durran, 1997]. Considering the influence of directional wind shear on the flow structures, further studies are needed on the wake vorticity and PV dynamics for turning flows, while the primary goals of this study is to document the very different behaviors of mountain gravity waves 
within directionally sheared flows for relatively high mountains or nonlinear flow regimes.

\section{Conclusions}

Numerical simulations were carried out to study the influence of directional wind shear on the momentum flux of three-dimensional (3D) gravity waves forced by circular bell-shaped mountains using the mesoscale nonhydrostatic ARPS model. The model base-state flow was dry, inviscid, and stably stratified. The Earth's rotation was omitted and for the given mountain width the flow was very close to being hydrostatic. Four directional wind profiles named W1, W2, W2a and W2b, with constant speed in the $x$ direction and linearly increasing speed with height in the $y$ direction were considered. At the surface the wind is westerly with W1 and northwesterly with W2. The speed of W1 increases with height whereas W2 experiences a wind speed decrease below $\sim 1.13 \mathrm{~km}$. W2a and W2b are akin to W2 except with a stronger vertical wind shear. In consequence, their speeds are only decelerated below $0.57 \mathrm{~km}$ and $0.4 \mathrm{~km}$, respectively. The wave momentum flux (WMF) was investigated for four different Froude numbers (Fr), corresponding to four different mountain heights (of 0.01, 0.5, 1 and $2 \mathrm{~km}$ ). Experiments with a constant wind profile having the same surface wind speed as the sheared-profiles were also run, for the four different mountain heights. The numerical results are consistent with those of earlier studies and provide references for comparisons with sheared profile cases.

For a $10 \mathrm{~m}$ high mountain for which $F r=80$, the waves are essentially linear. The simulated WMFs agree well with the linear theory, accounting for about $96.4 \%, 97.3 \%$ and $97.8 \%$ of their linear theoretical results at the surface for $\mathrm{W} 1$, W2, and W2a, respectively. This agreement remains good even at a low Richardson number of $\mathrm{Ri}=0.5$ where the surface WMF for W2b is increased by about 3.8\% in comparison to its analytic counterpart. These linear wave experiments thus provide a numerical validation for the theoretical study of $X W X 12$. For a moderately high mountain where $F r=1.6$, the model WMFs are qualitatively similar to their analytic counterparts, but are enhanced due to nonlinear effect. For high mountains of low Froude number, the height variation of the base-state wind speed comes into play, and has a pronounced influence on the WMF. The numerical model produces a "low-drag" WMF in W2 at $F r=0.8$, and increased WMFs (compared to linear theoretical prediction) in W1, W2a and W2b at $F r=0.4$, opposite to the case of constant flow at the same surface-based Froude number [MJ92; Eckermann et al., 2010].

Analysis on the mountain flow pattern shows that the increased speed of W1 acts to suppress lateral flow splitting. More air is allowed to climb over the mountain. On the contrary, W2 is more prone to flow around the mountain owing to its reduced speed in the below-mountain-peak layer. Although W2a and W2b are also firstly decelerated with height, the deceleration is confined to a shallow layer near the surface; above it their speeds change to increase with height rapidly such that they can also readily go over the mountain. It is also found that the wake flow pattern is asymmetric with respect to the direction of surface incoming wind, and exhibits a transition towards lee vortex shedding in $\mathrm{W} 2$ at $\mathrm{Fr}=0.4$.

To conclude, for turning flows, it is no longer appropriate to determine the drag state of the WMF on the basis of the surface Froude number only, because the WMF 
could differ distinctively even at the same Froude number. By affecting the way the flow goes over or around the mountain, the drag state of the nonlinear model solution can differ significantly from the prediction of linear theory, as far as changing from low to high drag states. By inducing asymmetric development of airflow in the mountain wake, the directional wind shear has the potential of triggering atmospheric vortex streets, significantly altering that drag state of the flow-over-mountain problem also. When vortex shedding occurs, steady state response can no longer be achieved. Due to the notable influence of directional wind shear on mountain waves, existing parameterization schemes for low-level mountain wave drag based on constant flow solutions are not be suitable for turning flows; new parameterization schemes taking into account of the turning effects need to be developed.

Because directionally sheared wind profiles and finite amplitude mountains are common in the real atmosphere, and mountain gravity waves and their momentum fluxes, as well as nonlinear flow responses and flow structures to the lee of mountains have significant impacts on the atmospheric circulations, more systematic studies on more general forms of the wind profiles and mountain shapes should be carried out in the future. In this study, only linearly increasing shear profiles and a circular bell-shaped mountain are considered; further we only examined forward wind shear. Teixeira et al. [2004] found in their numerical simulations that surface WMF decreased more rapidly with the Ri for forward-shear than backward-shear winds, and tentatively attributed this behavior to the non-hydrostatic effects. Understanding the dependency of the WMF on wind shear direction is another area needing further studies. Using the ERA-40 reanalysis wind profiles, Miranda et al. [2009] found that wind curvature effects were prominent in Antarctic and East Africa where easterly flows prevailed. Gravity wave drag (GWD) was enhanced significantly there, resulting in a reduction of the global westerly GWD torque. Therefore, future studies should also try to take into account of the wind profile curvature effects.

Acknowledgements. This work was primarily supported by the National 973 Fundamental Research Program of China (2013CB430103 and 2009CB421502), and by National Science Foundation of China grants 40775034, 40575017, 40830958. Xin Xu was supported by the Chinese Scholarship Council for his extended visit at the Center for Analysis and Prediction of Storms, University of Oklahoma. The third author acknowledges the support of the National Thousand Person Plan hosted at the Nanjing University, and the support of NSF grants AGS0802888, OCI-0905040, AGS-0941491, AGS-1046171, and AGS-1046081.

\section{References}

Aebischer, U., and C. Schär (1998), Low-level potential vorticity and cyclogenesis to the lee of the Alps. J. Atmos. Sci., 55, 186-207.

Clark, T. L., and W. R. Peltier (1984), Critical level reflection and the resonant growth of nonlinear mountain waves. J. Atmos. Sci., 41, 3122-3134.

Dörnbrack, A., J. D. Doyle, T. P. Lane, R. D. Sharman, and P. K. Smolarkiewicz (2005), On physical realizability and uncertainty of numerical solutions. Atmos. Sci. Lett., 6, 118-122, DOI: 10.1002/asl.100.

Doyle, J. D., and coauthors (2000), An intercomparison of model-predicted wave breaking for the 11 January 1972 Boulder windstorm. Mon. Wea. Rev., 129, 901914. 
Doyle, J. D., and coauthors (2011), An intercomparison of T-REX mountain wave simulations and implications for mesoscale predictability. Mon. Wea. Rev., 139, 2811-2831.

Durran, D. R., and J. B. Klemp (1987), Another look at downslope winds. Part II: Nonlinear amplification beneath wave-overturning layers. J. Atmos. Sci., 44, 34023412.

Eckermann, S. D., J. Lindeman, D. Broutman, J. Ma, and Z. Boybeyi (2010), Momentum fluxes of gravity waves generated by variable Froude number flow over threedimensional obsacles. J. Atmos. Sci., 67, 2260-2278.

Etling, D. (1989), On atmospheric vortex streets in the wake of large islands. Meteorol. Atmos. Phys., 41, 157-164.

Gregory, D., G. J. Shutts, and J. R. Mitchell (1998), A new gravity-wave-drag scheme incorporating anisotropic orography and low-level wave-breaking: Impact upon the climate of the UK Meteorological Office Unified Model. Quart. J. Roy. Meteor. Soc., 124, 463-493.

Grubišić, V. (2004), Bora-driven potential vorticity banners over the Adriatic. Quart. J. Roy. Meteor. Soc., 130, 2571-2603.

Grubišić, V., R. B. Smith, and C. Schär (1995), The effect of bottom friction on shallowwater flow past an isolated obstacle. J. Atmos. Sci., 52, 1985-2005.

Hunt, C. R., and W. H. Snyder (1980), Experiments on stably and neutrally stratified flow over a model three-dimensional hill. J. Fluid Mech., 96, 671-704.

Jiang, Q., R. B. Smith, and J. Doyle (2003), The nature of the mistral: Observations and modeling of two MAP events. Quart. J. Roy. Meteor. Soc., 129, 857-875.

Kim, Y. J., and A. Arakawa (1995), Improvement of orographic gravity wave parameterization using a mesoscale gravity wave model. J. Atmos. Sci., 52, 18751902.

Kim, Y. J., and J. Doyle (2005), Extension of an orographic-drag parameterization scheme to incorporate orographic anisotropy and flow blocking. Quart. J. Roy. Meteor. Soc., 131, 1893-1921.

Kim, Y. J., S. D. Eckermann, and H. Y. Chun (2003), An overview of the past, present and future of gravity-wave drag parameterization for numerical climate and weather prediction models. Atmos. - ocean, 41, 65-98.

Lott, F., and M. Miller (1997), A new sub-grid orographic drag parameterization: Its formulation and testing. Quart. J. Roy. Meteor. Soc., 123, 101-127.

Miranda, P. M. A., and I. N. James (1992), Non-linear three-dimensional effects on gravity-wave drag: Splitting flow and breaking waves. Quart. J. Roy. Meteor. Soc., 118, 1057-1081.

Miranda, P. M. A., and M. A. Valente (1997), Critical level resonance in threedimensional flow past isolated mountains. J. Atmos. Sci., 54, 1574-1588.

Miranda, P. M. A., J. P. A. Martins, and M. A. C. Teixeira (2009), Assessing wind profile effects on the global atmospheric torque. Quart. J. Roy. Meteor. Soc., 135, 807-814.

Rotunno, R., V. Grubišić, and P. K. Smolarkiewicz (1999), Vorticity and potential vorticity in mountain wakes. J. Atmos. Sci., 56, 2796-2810.

Schär, C. (1993), A generalized Bernoulli’s theorem. J. Atmos. Sci., 50, 1437-1443.

Schär, C., and D. R. Durran (1997), Vortex formation and vortex shedding in continuously stratified flows past isolated topography. J. Atmos. Sci., 54, 534-554. 
Schär, C., and R. B. Smith (1993a), Shallow water flow past isolated topography. Part I: Vorticity production and wake formation. J. Atmos. Sci., 50, 1373-1400.

Schär, C., and R. B. Smith (1993b), Shallow water flow past isolated topography. Part II: Transition to vortex shedding. J. Atmos. Sci., 50, 1401-1412.

Schär, C., M. Sprenger, D. Lüthi, Q. Jiang, R. B. Smith, and R. Benoit (2003), Structure and dynamics of an Alpine potential-vorticity banner. Quart. J. Roy. Meteor. Soc., 129, 825-855.

Scinocca, J. F., and N. A. McFarlane (2000), The parameterization of drag induced by stratified flow over anisotropic orography. Quart. J. Roy. Meteor. Soc., 126, 23532393.

Shutts, G. J. (1995), Gravity-wave drag parametrization over complex terrain: The effect of critical level absorption in directional wind-shear. Quart. J. Roy. Meteor. Soc., 121, 1005-1021.

Shutts, G. J. (1998), Stationary gravity-wave structure in flows with directional wind shear. Quart. J. Roy. Meteor. Soc., 124, 1421-1442.

Smith, R. B. (1980), Linear theory of stratified hydrostatic flow past an isolated mountain. Tellus, 32, 348-364.

Smith, R. B. (1985), On severe downslope winds. J. Atmos. Sci., 42, 2597-2603.

Smith, R. B. (1989), Hydrostatic airflow over mountains. Advances in Geophysics, Vol. 31, Academic Press, 1-41.

Smith, R. B., and S. Grønås (1993), Stagnation points and bifurcation in 3-D mountain airflow. Tellus, 45, 28-43.

Smolarkiewicz, P. K., and R. Rotunno (1989), Low Froude number flow past three dimensional obstacles. Part I: Baroclinically generated lee vortices. J. Atmos. Sci., 46, 1154-1164.

Sun, W. Y., and J. D. Chern (1994), Numerical experiments of vortices in the wakes of large idealized mountains. J. Atmos. Sci., 51, 191-209.

Teixeira, M. A. C., P. M. A. Miranda, and M. A. Valente (2004), An analytical model of mountain wave drag for wind profiles with shear and curvature. J. Atmos. Sci., 61, 1040-1054.

Teixeira, M. A. C., and P. M. A. Miranda (2009), On the momentum fluxes associated with mountain waves in directionally sheared flows. J. Atmos. Sci., 66, 3419-3433.

Thorpe, A. J., H. Volkert, and D. Heimman (1993), Potential vorticity of flow along the Alps. J. Atmos. Sci., 50, 1573-1590.

Vosper, S. B. (2000), Three-dimensional numerical simulations of strongly stratified flow past conical orography. J. Atmos. Sci., 57, 3716-3739.

Wang, Q., and Z. Tan (2009), Idealized numerical simulation study of the potential vorticity banners over a mesoscale mountain: Dry adiabatic process. Adv. Atmos. Sci., 26, 906-922.

Xu, X., Y. Wang, and M. Xue (2012), Momentum flux and flux divergence of gravity waves in directional shear flows over three-dimensional mountains. J. Atmos. Sci.,in press.

Xue, M., K. K. Droegemeier, and V. Wong (2000), The Advanced Regional Prediction System (ARPS) - A multi-scale nonhydrostatic atmospheric simulation and prediction model. Part I: Model dynamics and verification. Meteorol. Atmos. Phys., 75, 161-193. 


\section{List of Figures}

Fig. 1. Vertical profiles of model simulated WMF magnitude at non-dimensional time $T$ = 72 in ExpCST1 (thick solid), ExpCST2 (thin solid), ExpCST3 (dashed), and ExpCST4 (dotted). All WMFs are normalized by the momentum fluxes of corresponding linear theoretical hydrostatic mountain wave solutions of constant flow with the same surface speed, as given by Eq. (3).

Fig. 2. Vertical profiles of model simulated WMF magnitude at non-dimensional time $T$ = 43.2 in (a) Exp1 (dashed), Exp1a (dotted), Exp1b (long-dashed), (b) Exp2 (dashed), (c) Exp2a (dashed), and (d) Exp2b (dashed). Solid lines are obtained from linear wave theory as given by Eq. (4). All WMFs are normalized by the momentum flux of linear hydrostatic waves of constant flow with the same surface speed, as given by Eq. (3).

Fig. 3. Vertical profiles of the magnitude of simulated WMF divergence (solid circle) at $T$ = 43.2 from (a) Exp1, (b) Exp2, (c) Exp2a and (d) Exp2b, as compared to corresponding analytical profiles (solid line).

Fig. 4. Vertical profiles of WMF magnitudes at $T=100.8$ in (a) Exp3, Exp5, Exp7, (b) Exp4, Exp6, Exp8, (c) Exp4a, Exp6a, Exp8a, and (d) Exp4b, Exp6b, Exp8b. Solid lines are for linear wave theory, open circles are for $\mathrm{Fr}=1.6$ (Exp3, Exp4, Exp4a, Exp4b), solid circles are for $F r=0.8$ (Exp5, Exp6, Exp6a, Exp6b), and triangles are for $\mathrm{Fr}=0.4$ (Exp7, Exp8, Exp8a, Exp8b).

Fig. 5. Same as Fig. 4 but for the WMF divergence in (a) Exp3, Exp5, Exp7, (b) Exp4, Exp6, Exp8, (c) Exp4a, Exp6a, Exp8a, and (d) Exp4b, Exp6b, Exp8b. All the WMF divergences are scaled by a factor of $\left(0.01 / h_{0}\right)^{2}$ where $h_{0}$ is the mountain height in $\mathrm{km}$.

Fig. 6. Surface wind vectors at $T=100.8$ in (a) Exp5, (b) Exp6, (c) Exp6a, (d) Exp6b, (e) Exp7, (f) Exp8, (g) Exp8a, and (h) Exp8b. Solid circles represent the contour of half the mountain height. Straight gray lines indicate the positions of vertical cross-sections shown in Fig. 7.

Fig. 7. Isentropes in the vertical cross-section along the direction of surface wind (i.e. gray lines in Fig. 6) in (a) Exp5, (b) Exp6, (c) Exp6a, (d) Exp6b, (e) Exp7, (f) Exp8, (g) Exp8a, and (h) Exp8b at $T=100.8$.

Fig. 8. Temporal evolution of scaled surface pressure drag in Exp8. The normalization is with respect to that in constant flows given by Eq. (3).

Fig. 9. Vertical vorticity at the surface in Exp8 at (a) $T=7.2$, (b) $T=14.4$, (c) $T=21.6$, (d) $T=28.8$, (e) $T=36.0$ and (f) $T=43.2$. Solid circle represents the contour of half the mountain height. Dashed contours are negative. 
Table 1. List of numerical experiments

\begin{tabular}{llcccc}
\hline Experiment & $\begin{array}{c}\text { Wind } \\
\text { profile }\end{array}$ & $\begin{array}{c}\text { Domain } \\
\text { width } \\
(L, \mathrm{~km})\end{array}$ & $\begin{array}{c}\text { Domain } \\
\text { depth } \\
\left(H_{0}, \mathrm{~km}\right)\end{array}$ & $\begin{array}{c}\text { Mountain } \\
\text { height } \\
\left(h_{0}, \mathrm{~km}\right)\end{array}$ & $\begin{array}{c}\text { Surface-based } \\
\text { Froude number } \\
(F r)\end{array}$ \\
\hline ExpCST1 & W0 & $1440\left(L_{0}\right)$ & 16 & 0.01 & 80 \\
ExpCST2 & W0 & $L_{0}$ & 16 & 0.5 & 1.6 \\
ExpCST3 & W0 & $L_{0}$ & 16 & 1.0 & 0.8 \\
ExpCST4 & W0 & $L_{0}$ & 16 & 2.0 & 0.4 \\
Exp1 & W1 & $L_{0}$ & 16 & 0.01 & 80 \\
Exp1a & W1 & $1.25 L_{0}$ & 16 & 0.01 & 80 \\
Exp1b & W1 & $1.5 L_{0}$ & 16 & 0.01 & 80 \\
Exp2 & W2 & $L_{0}$ & 16 & 0.01 & 80 \\
Exp2a & W2a & $L_{0}$ & 8 & 0.01 & 80 \\
Exp2b & W2b & $L_{0}$ & 6 & 0.01 & 80 \\
Exp3 & W1 & $L_{0}$ & 16 & 0.5 & 1.6 \\
Exp4 & W2 & $L_{0}$ & 16 & 0.5 & 1.6 \\
Exp4a & W2a & $L_{0}$ & 8 & 0.5 & 1.6 \\
Exp4b & W2b & $L_{0}$ & 6 & 0.5 & 1.6 \\
Exp5 & W1 & $L_{0}$ & 16 & 1.0 & 0.8 \\
Exp6 & W2 & $L_{0}$ & 16 & 1.0 & 0.8 \\
Exp6a & W2a & $L_{0}$ & 8 & 1.0 & 0.8 \\
Exp6b & W2b & $L_{0}$ & 6 & 1.0 & 0.8 \\
Exp7 & W1 & $L_{0}$ & 16 & 2.0 & 0.4 \\
Exp8 & W2 & $L_{0}$ & 16 & 2.0 & 0.4 \\
Exp8a & W2a & $L_{0}$ & 8 & 2.0 & 0.4 \\
Exp8b & W2b & $L_{0}$ & 6 & 2.0 & 0.4 \\
Exp8U & W2U & $L_{0}$ & 16 & 2.0 & 0.4 \\
\hline
\end{tabular}


Table 2. List of wind profiles adopted in numerical experiments

\begin{tabular}{llccccc}
\hline \multicolumn{1}{c}{ Wind type } & $\begin{array}{l}\text { Wind } \\
\text { profile }\end{array}$ & $\begin{array}{c}U_{0} \\
\left(\mathrm{~m} \mathrm{~s}^{-1}\right)\end{array}$ & $\begin{array}{c}V_{0} \\
\left(\mathrm{~m} \mathrm{~s}^{-1}\right)\end{array}$ & $\begin{array}{c}U_{Z} \\
\left(\mathrm{~s}^{-1}\right)\end{array}$ & $\begin{array}{c}V_{Z} \\
\left(\mathrm{~s}^{-1}\right)\end{array}$ & $\begin{array}{c}\text { Richardson } \\
\text { number (Ri) }\end{array}$ \\
\hline Constant wind & W0 & 8 & 0 & 0 & 0 & $\infty$ \\
Directional & W1 & 8 & 0 & 0 & $5 \times 10^{-3}$ & 4 \\
shear wind & W2 & $4 \sqrt{2}$ & $-4 \sqrt{2}$ & 0 & $5 \times 10^{-3}$ & 4 \\
& W2a & $4 \sqrt{2}$ & $-4 \sqrt{2}$ & 0 & $10^{-2}$ & 1 \\
& W2b & $4 \sqrt{2}$ & $-4 \sqrt{2}$ & 0 & $\sqrt{2} \times 10^{-2}$ & 0.5 \\
$\begin{array}{l}\text { Unidirectional } \\
\text { shear wind }\end{array}$ & W2U & 8 & 0 & \multicolumn{2}{c}{ speed matching that of W2 } \\
\hline
\end{tabular}




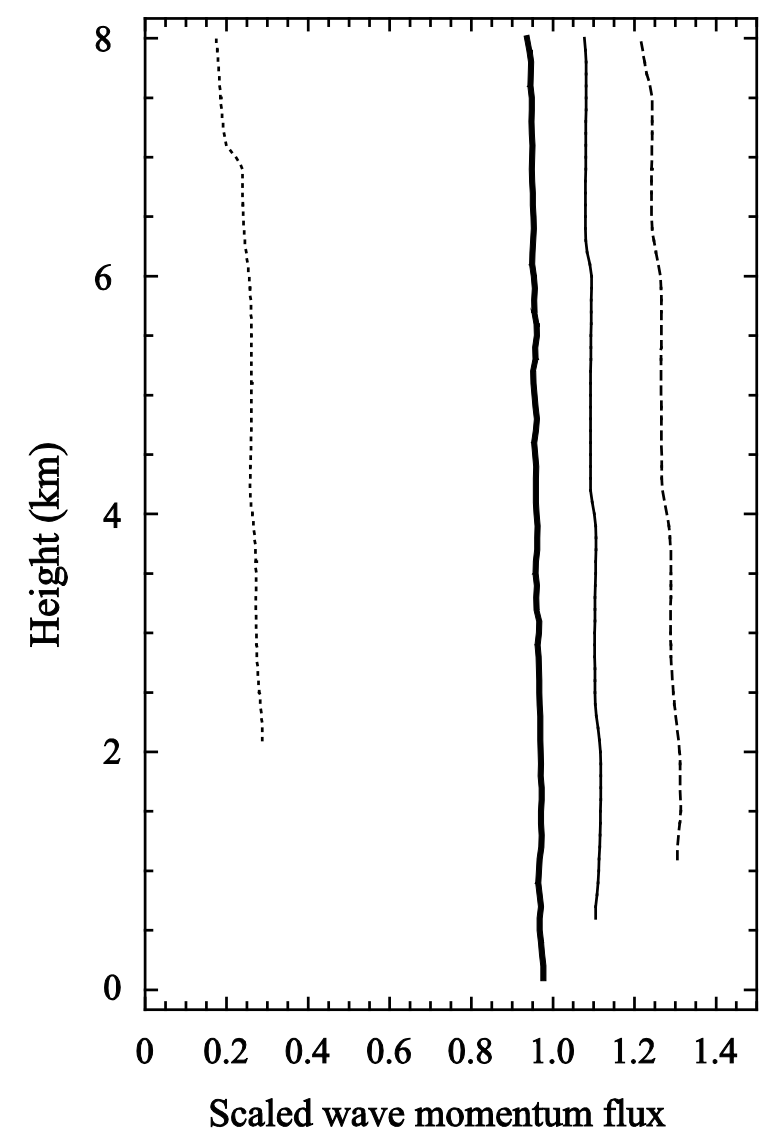

Fig. 1. Vertical profiles of model simulated WMF magnitude at non-dimensional time $T$ = 72 in ExpCST1 (thick solid), ExpCST2 (thin solid), ExpCST3 (dashed), and ExpCST4 (dotted). All WMFs are normalized by the momentum fluxes of corresponding linear theoretical hydrostatic mountain wave solutions of constant flow with the same surface speed, as given by Eq. (3). 

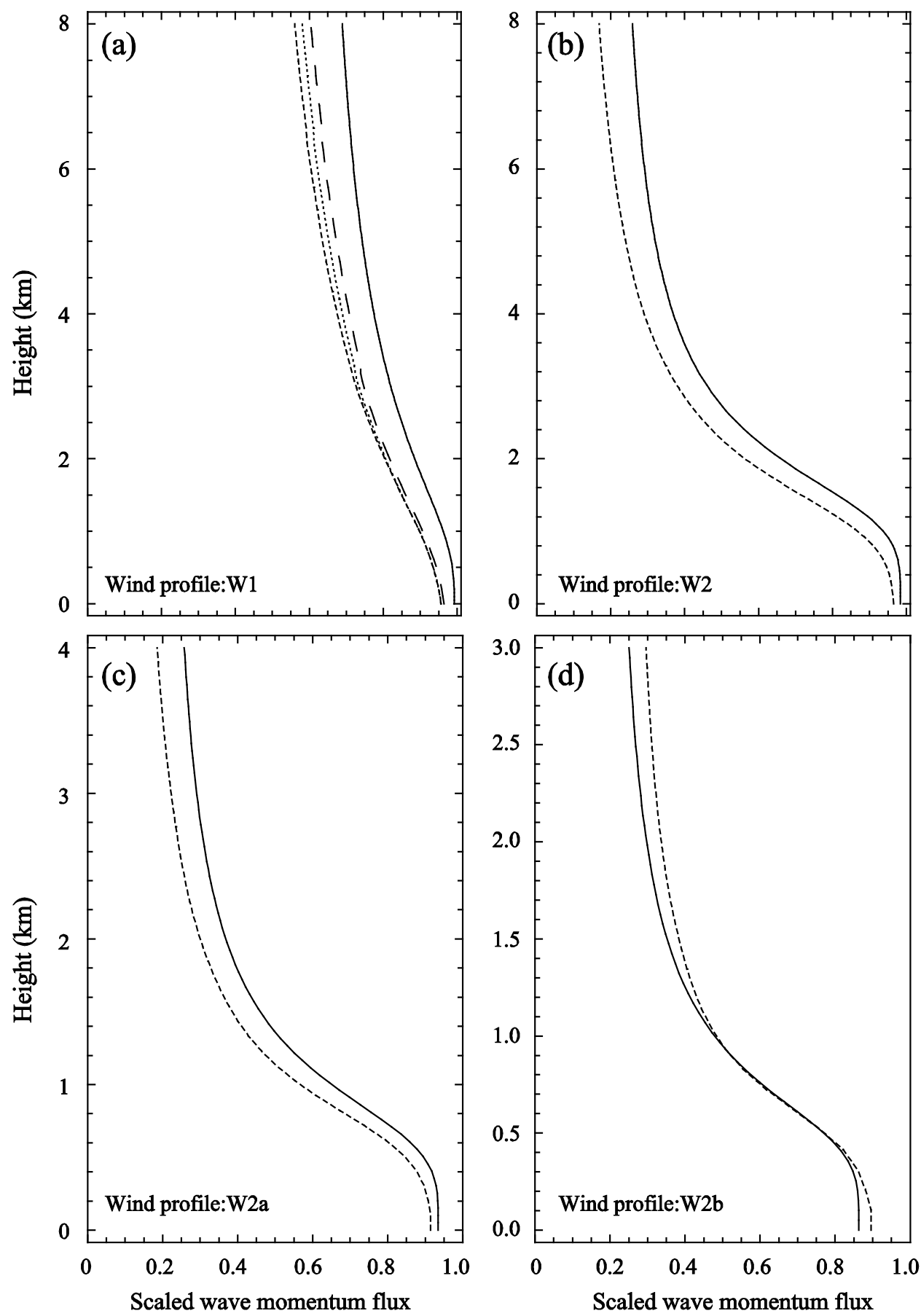

Fig. 2. Vertical profiles of model simulated WMF magnitude at non-dimensional time $T$ = 43.2 in (a) Exp1 (dashed), Exp1a (dotted), Exp1b (long-dashed), (b) Exp2 (dashed), (c) Exp2a (dashed), and (d) Exp2b (dashed). Solid lines are obtained from linear wave theory as given by Eq. (4). All WMFs are normalized by the momentum flux of linear hydrostatic waves of constant flow with the same surface speed, as given by Eq. (3). 

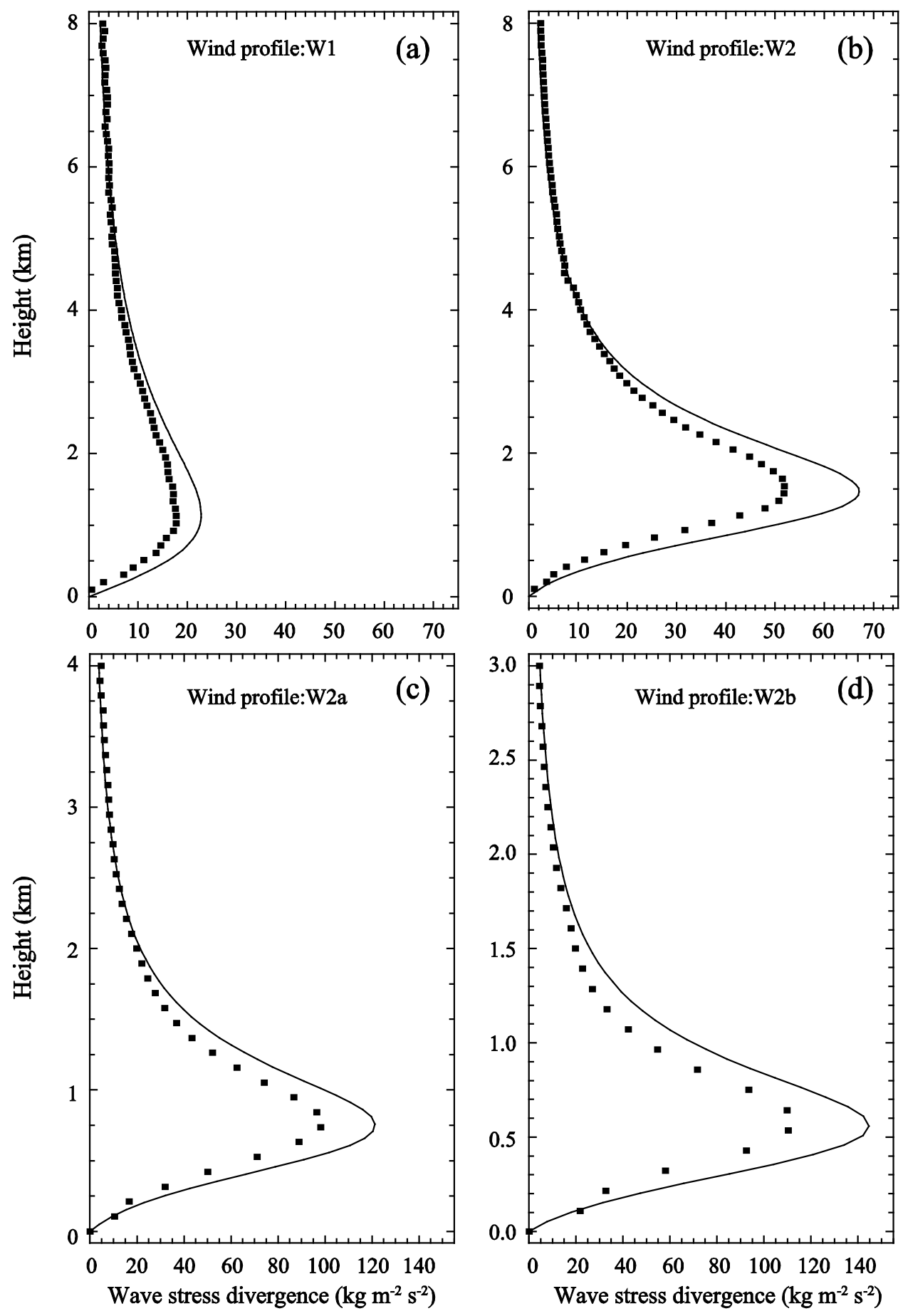

Fig. 3. Vertical profiles of the magnitude of simulated WMF divergence (solid circle) at $T$ = 43.2 from (a) Exp1, (b) Exp2, (c) Exp2a and (d) Exp2b, as compared to corresponding analytical profiles (solid line). 

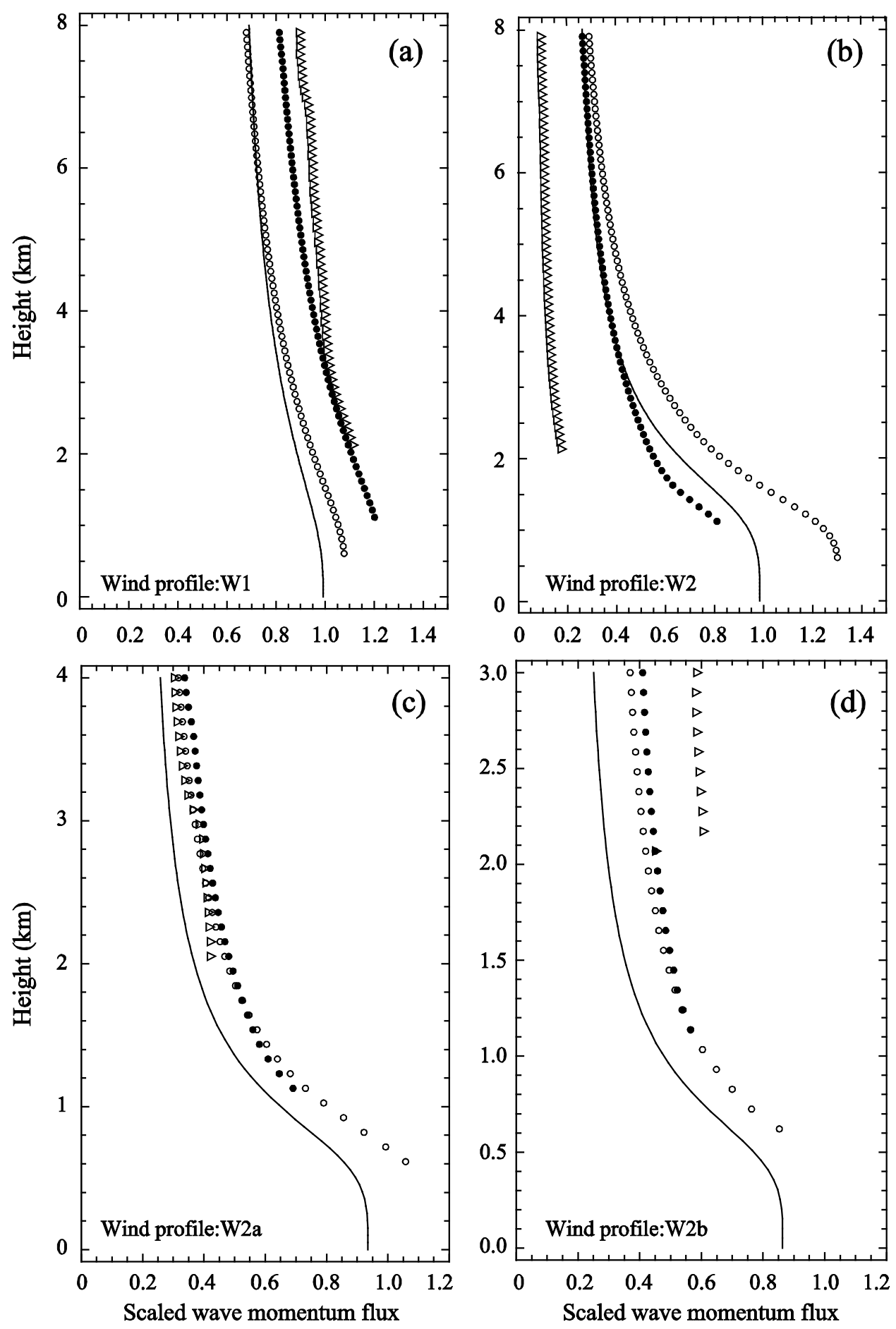

Fig. 4. Vertical profiles of WMF magnitudes at $T=100.8$ in (a) Exp3, Exp5, Exp7, (b) Exp4, Exp6, Exp8, (c) Exp4a, Exp6a, Exp8a, and (d) Exp4b, Exp6b, Exp8b. Solid lines are for linear wave theory, open circles are for $F r=1.6$ (Exp3, Exp4, Exp4a, Exp4b), solid circles are for $F r=0.8$ (Exp5, Exp6, Exp6a, Exp6b), and triangles are for $F r=0.4$ (Exp7, Exp8, Exp8a, Exp8b). 

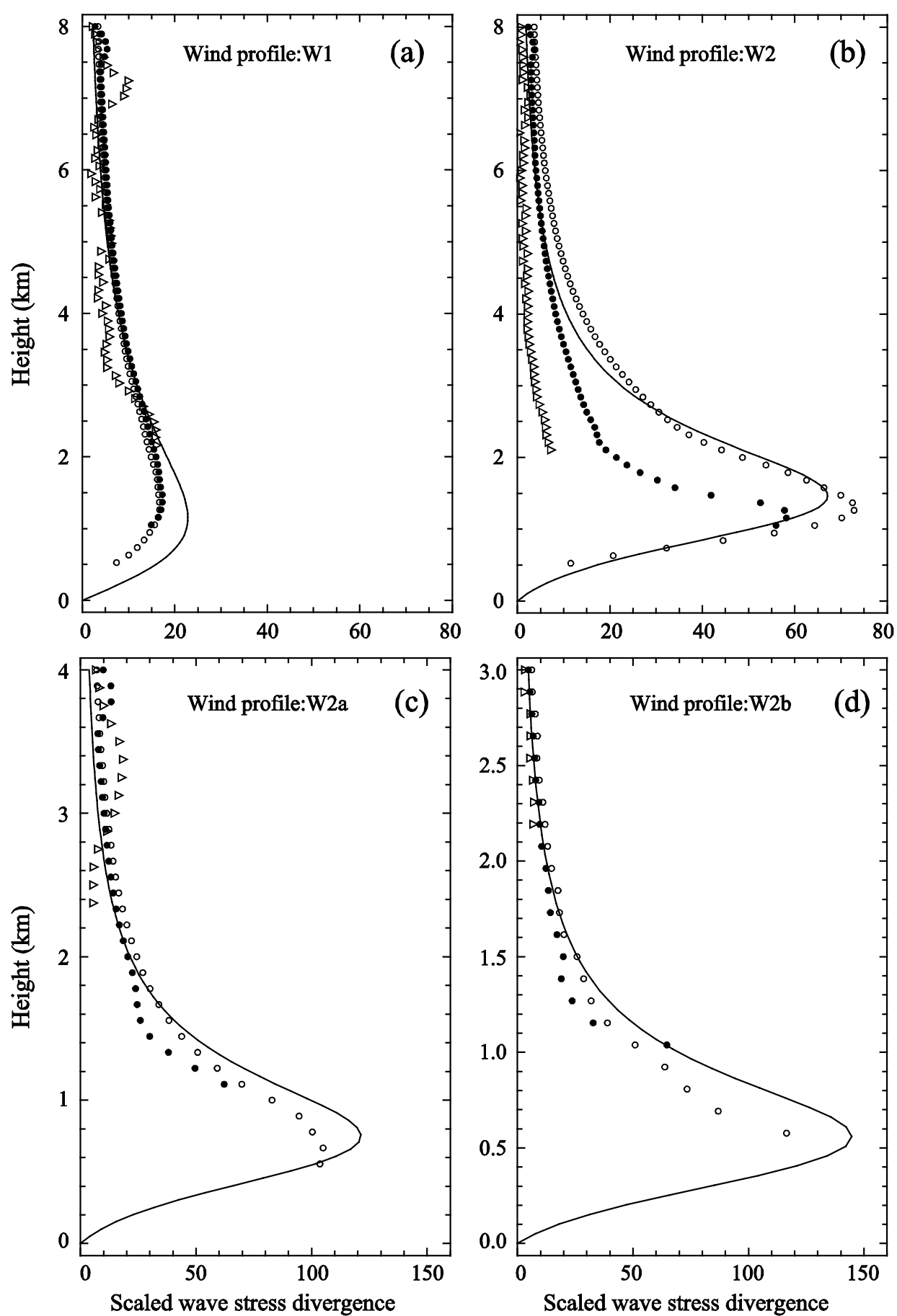

Fig. 5. Same as Fig. 4 but for the WMF divergence in (a) Exp3, Exp5, Exp7, (b) Exp4, Exp6, Exp8, (c) Exp4a, Exp6a, Exp8a, and (d) Exp4b, Exp6b, Exp8b. All the WMF divergences are scaled by a factor of $\left(0.01 / h_{0}\right)^{2}$ where $h_{0}$ is the mountain height in $\mathrm{km}$. 

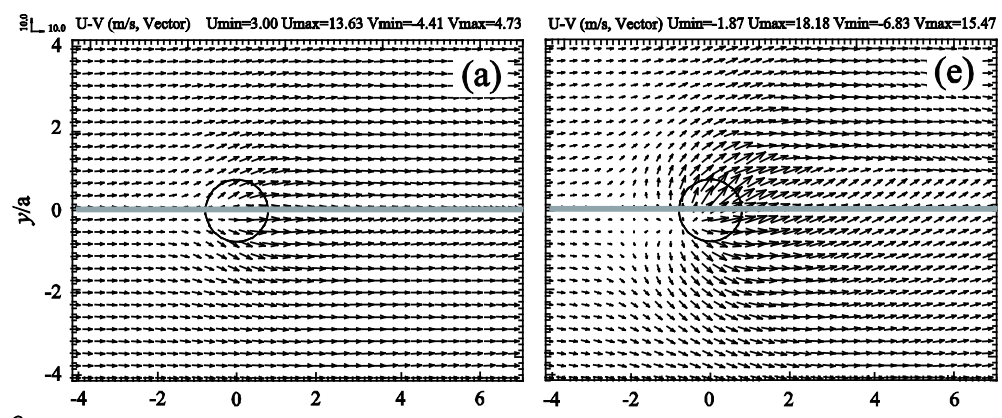

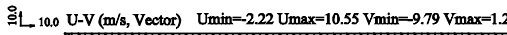
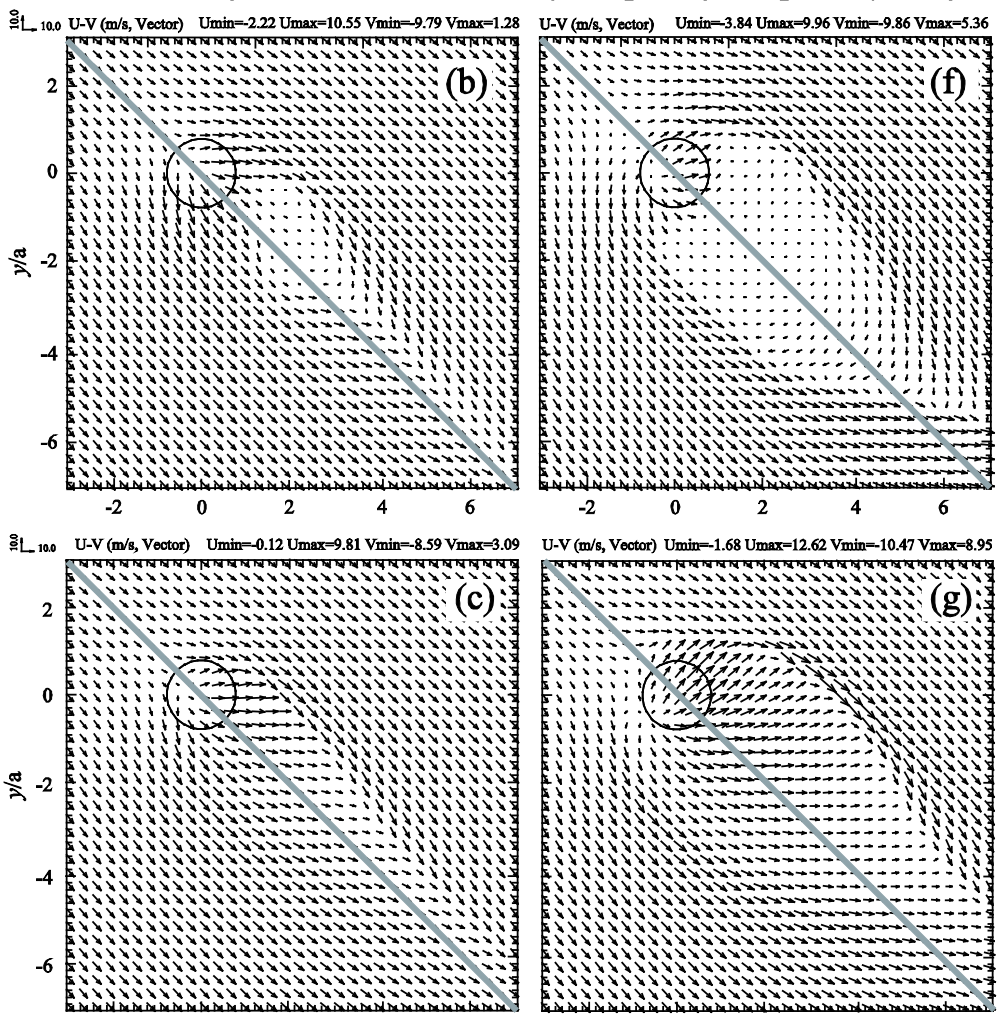

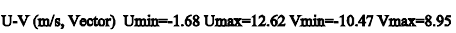
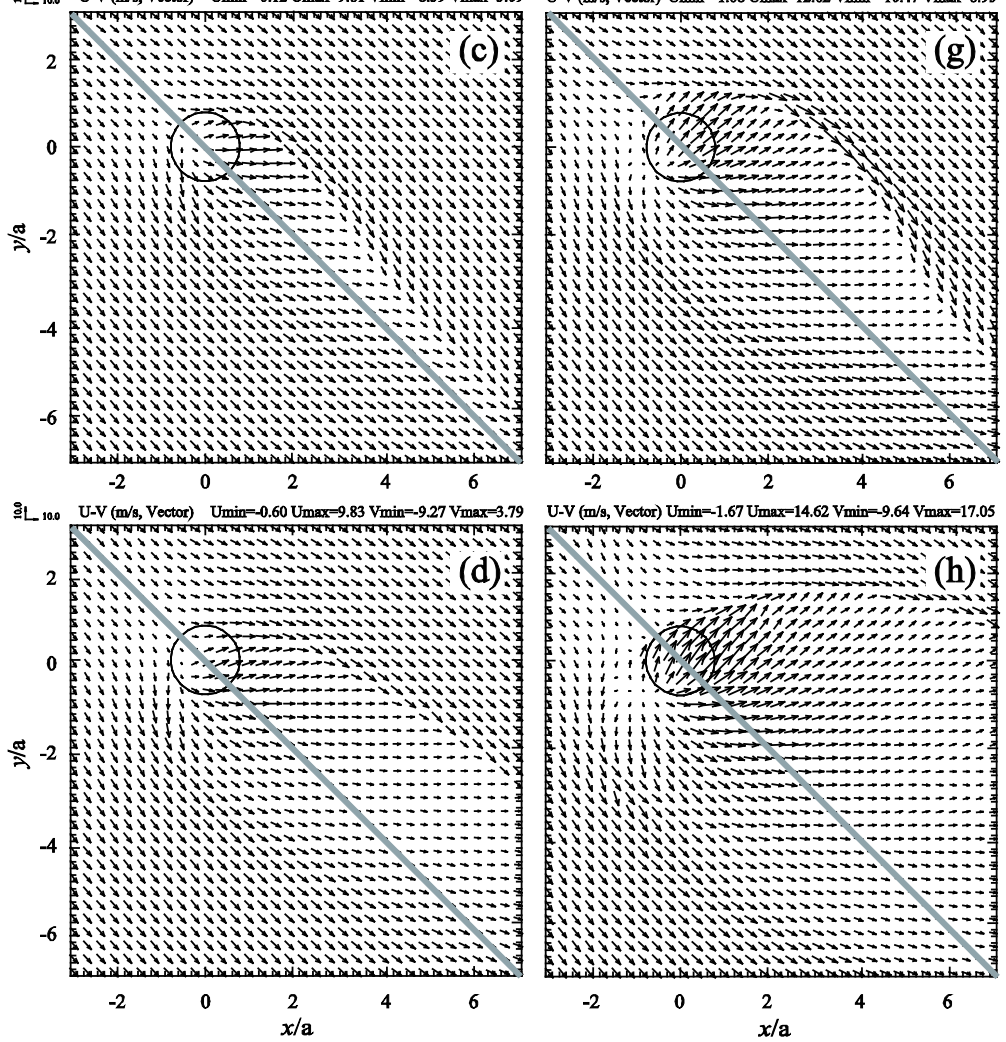

Fig. 6. Surface wind vectors at $T=100.8$ in (a) Exp5, (b) Exp6, (c) Exp6a, (d) Exp6b, (e) Exp7, (f) Exp8, (g) Exp8a, and (h) Exp8b. Solid circles represent the contour of half the mountain height. Straight gray lines indicate the positions of vertical cross-sections shown in Fig. 7. 

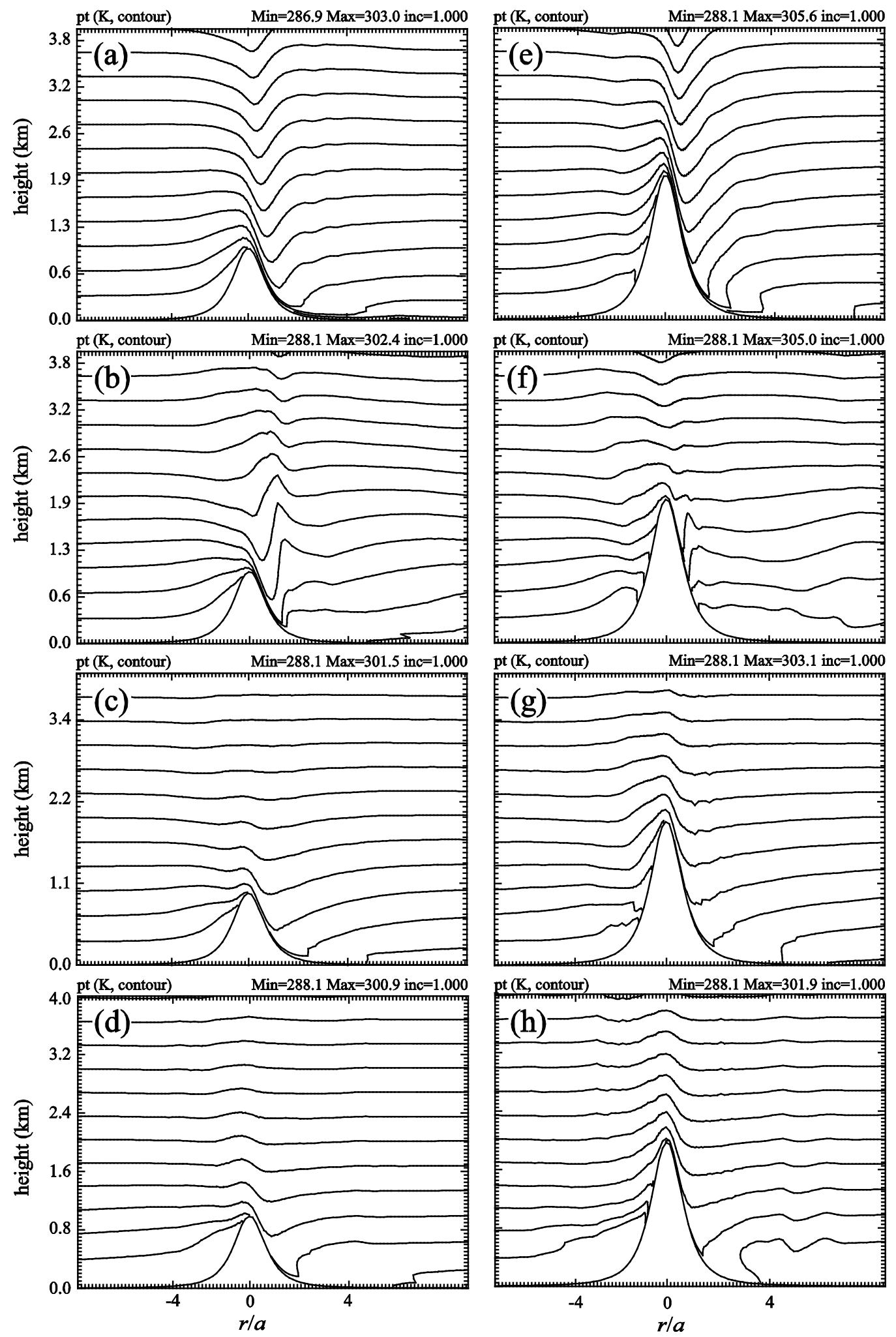

Fig. 7. Isentropes in the vertical cross-section along the direction of surface wind (i.e. gray lines in Fig. 6) in (a) Exp5, (b) Exp6, (c) Exp6a, (d) Exp6b, (e) Exp7, (f) Exp8, (g) Exp8a, and (h) Exp8b at $T=100.8$. 


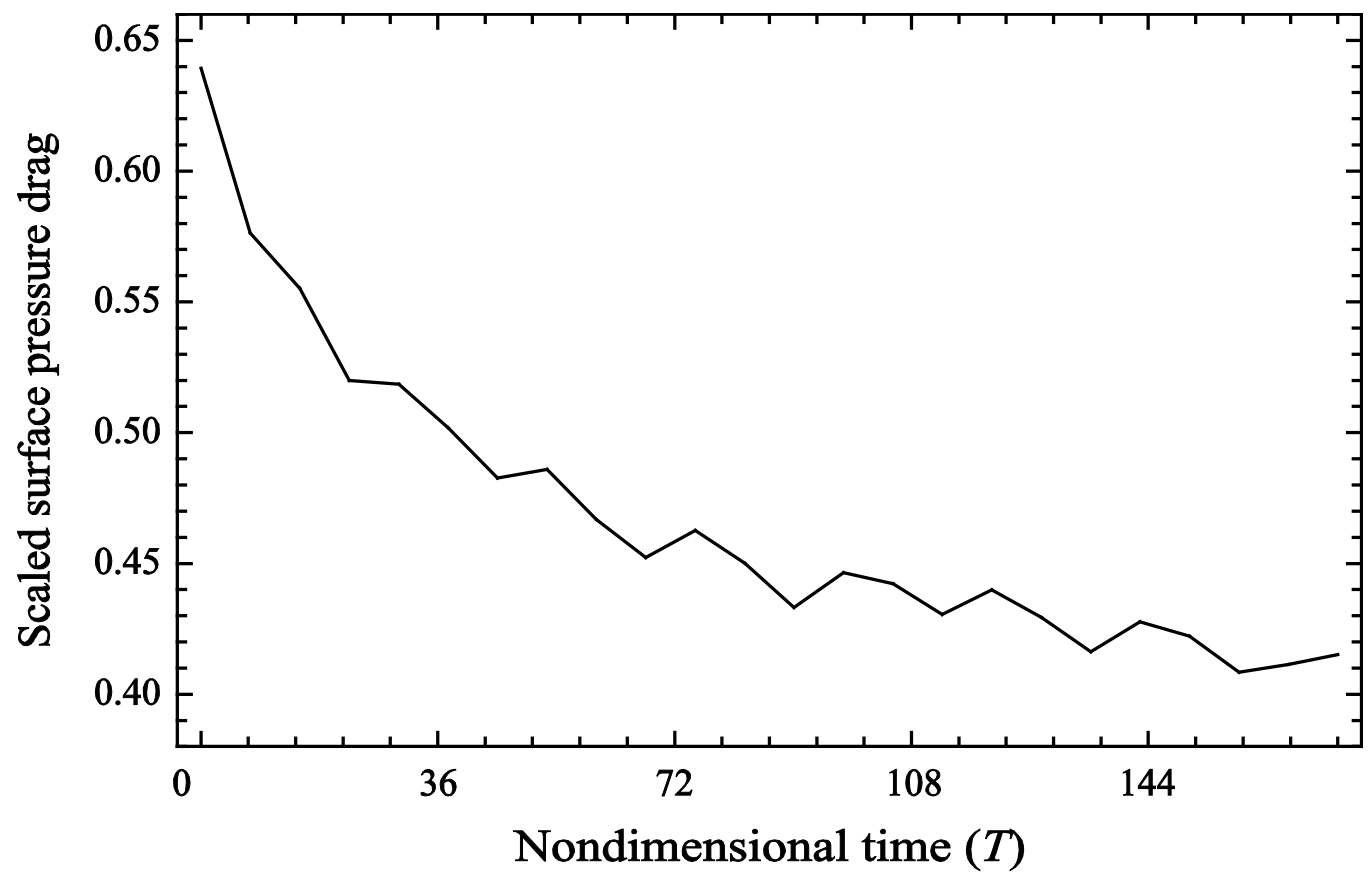

Fig. 8. Temporal evolution of scaled surface pressure drag in Exp8. The normalization is with respect to that in constant flows given by Eq. (3). 

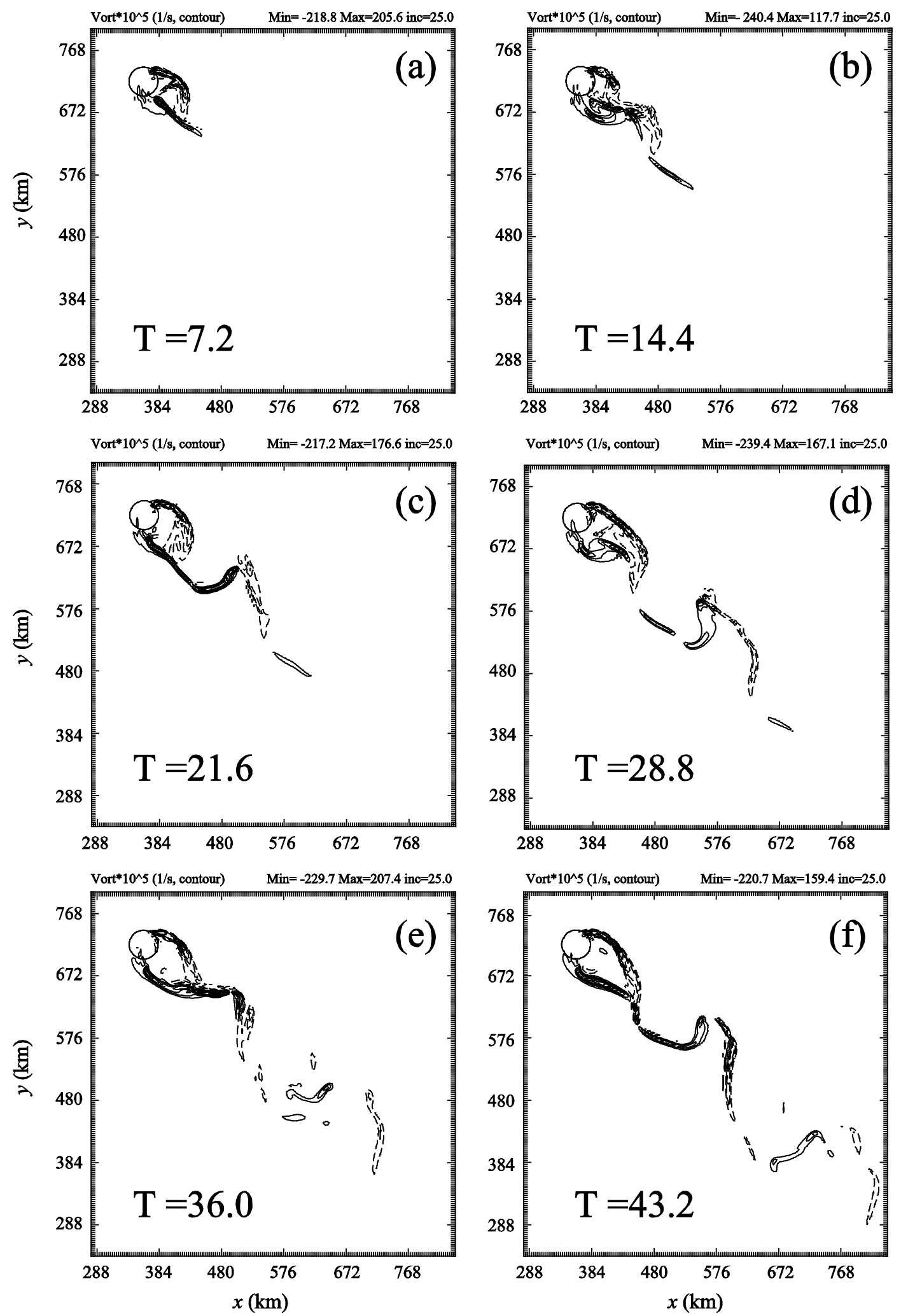

Fig. 9. Vertical vorticity at the surface in Exp8 at (a) $T=7.2$, (b) $T=14.4$, (c) $T=21.6$, (d) $T=28.8$, (e) $T=36.0$ and (f) $T=43.2$. Solid circle represents the contour of half the mountain height. Dashed contours are negative. 